\title{
SERVO CONSTRAINT CONTROL FOR MECHANICAL SYSTEMS: FRICTION FORCE DEPENDING ON CONTROL DESIGN
}

\author{
QINGMIN HUANG \\ SAIC GM, Wuling Automobile Co., Ltd., Liuzhou, Guangxi, China \\ e-mail: qingmin.huang@sgmw.com.cn \\ JIN HUANG \\ School of Vehicle and Mobility, Tsinghua University, China \\ e-mail: huangjin@tsinghua.edu.cn (corresponding author)
}

\begin{abstract}
In this paper, the problem of motion of controlled mechanical systems under a servo constraint is considered. The servo constraint which is prescribed by the designer is supposed to be non-ideal, that is, it does work in a virtual displacement. The second order form constraint is introduced to obtain a closed-form (i.e., analytical form) control input. The final servo control contains two parts: the first one generates the constraint force so that the constraint is exactly followed, while the second one can be designed by the designer for the facility, such as to compensate the effects of the friction force. After geometrical analysis applied to the Coulomb friction forces, we found that they actually depend on the control forces (i.e., the two are coupled). Application to a 3-DOF robot manipulator is made.
\end{abstract}

Keywords: mechanical systems, modeling, non-ideal constraints, motion control

\section{Introduction}

A mechanical system is often required to follow certain constraints. Sometimes these constraints are fulfilled by constraint forces generated by the Nature. That is, when one determines motion of a system, it is generally assumed that the environment (which includes the structure of the machines) can automatically generate the required constraint force. Those constraints are called passive constraints (i.e., material constraints) as the constraint is followed in a passive manner. This passive constraint problem is along with the original Lagrange aspect. Many important contributions have been made on the subject (for a survey, see, for example, Papastavridis, 2002).

In contrast to this, the servo constraint problem is to find what the engineer should do, so that the constraints are followed. This problem is one of the few frontiers in analytical mechanics that have rarely been studied until recently. In the past, efforts on this problem were made on individual examples (see, e.g., Cabannes, 1968; Kirgetov, 1967; Zhao et al., 2020; Huang et al., 2013) instead of a systematic framework. One reason is perhaps the concepts of servos (together with implementation via use of microprocessors) is relatively new, in comparison to other developments in analytical mechanics. Chen (2009) proposed a systematic approach for the servo constraint problem. In this paper, we followed the work by Chen (2009) and considered the case when the servo constraints are non-ideal. For example, when friction forces exist in the mechanical system. Following Udwadia and Kalaba (1996), the Moore-Penrose inverse of a matrix is adopted to explore the geometric structure of the constraints. Provided certain assumptions are met, the analytical expression of the servo control is obtained. After geometrical analysis of the Coulomb friction force, it is very interesting to find out that the friction force is actually coupled with the control force. This is really helpful since in some textbooks the friction 
force can be compensated by the control forces, see Sciavicco and Siciliano (2000), in which even the normal force is taken to be constant.

The primary objective of this article is to develop a method to obtain a closed-form control force in a mechanical system under an ideal or non-ideal servo constraint. The control force keeps the mechanical system strictly constrained and can compensate the effect of friction.

The main contributions of the article are threefold. Firstly, the non-ideal servo constraint problem is formulated. The second order form constraint which contains the acceleration explicitly and linearly is used to describe the servo constraint. Both holonomic and nonholonomic constraints can be written in this form. Because of the linearity in the acceleration, the Moore-Penrose inverse of a matrix can be adopted to explore the geometric structure of the constraints.

Secondly, the servo control for the non-ideal constraint is obtained. The servo control consists of two parts: the first one generates the constraint force, while the second one is an extra force that can be designed to compensate the friction force. The servo control is in an analytical form (i.e., closed-form). This control turns out to be a model-based state feedback control, and is readily applicable. No auxiliary variables such as Lagrange multipliers or pseudo-generalized speeds are needed. Besides, the control is continuous in the state.

Thirdly, the friction force is found to be coupled with the control force. A geometrical analysis is applied to the Coulomb friction force, in which it turns out that the Coulomb friction force is based on the specific control applied. This is a surprising outcome, which defies some conventional wisdom in which it was believed that the Coulomb friction force can be determined a priori of the control design.

The rest of the paper is outlined as follows. Section 2 provides a brief introduction about the passive ideal constraint problem. Section 3 presents the modeling of the servo constraint problem. The Moore-Penrose inverse is detailed in Section 4 as preliminaries before designing the detailed control law. Section 5 proposes the servo constraint control for the above mechanical system. Section 6 discusses the non-ideal constraint case. Section 7 illustrates the proposed servo control. Finally, conclusions are made in Section 8.

\section{Passive ideal constraint problem}

Consider a discrete mechanical system whose configuration can be described by $n$ dimensional generalized coordinates $\mathbf{q}=\left[q_{1}, q_{2}, \ldots, q_{n}\right]^{\mathrm{T}}$. The kinetic energy $T=T(\mathbf{q}, \dot{\mathbf{q}}, t)$ of the system, relative to an inertial frame, takes the form (Rosenberg, 1977)

$$
T(\mathbf{q}, \dot{\mathbf{q}}, t)=\frac{1}{2} \dot{\mathbf{q}}^{\mathrm{T}} \mathbf{M}(\mathbf{q}, t) \dot{\mathbf{q}}+\mathbf{N}(\mathbf{q}, t) \dot{\mathbf{q}}+\mathbf{P}(\mathbf{q}, t)
$$

where $\mathbf{M}(\mathbf{q}, t)=\mathbf{M}^{\mathrm{T}}(\mathbf{q}, t) \in \mathbb{R}^{n \times n}, \mathbf{N}(\mathbf{q}, t) \in \mathbb{R}^{1 \times n}$, and $\mathbf{P}(\mathbf{q}, t) \in \mathbb{R}^{n}$. Here the three terms on the right-hand side are contributions due to quadratic, linear and constant terms of $\dot{\mathbf{q}}$, respectively.

Suppose that the system is under $m$ passive (i.e., material) ideal constraints

$$
\sum_{i=1}^{n} A_{l i}(\mathbf{q}, t) \dot{q}_{i}+A_{l}(\mathbf{q}, t)=0 \quad l=1, \ldots, m
$$

where $A_{l i}(\cdot)$ and $A_{l}(\cdot)$ are both $C^{1}, m \leqslant n$. The constraints may not be integrable (and hence nonholonomic). The $\dot{\mathbf{q}}$ satisfying equation (2.2) is called the possible velocity.

Remark. By an ideal constraint, we mean that the constraint force which is induced by the constraint does no work in a virtual displacement. This is a basic assumption in classical analytical mechanics. However, this has been generalized to the non-ideal constraint force 
which will do work in a virtual displacement (Udwadia and Kalaba, 2000; Sun et al., 2015). Such a constraint force may occur when there is a sliding friction or some electromagnetic interaction occurs in the system.

The Pfaffian form of equation (2.2) is given by Abraham et al. (1988) as

$$
\sum_{i=1}^{n} A_{l i}(\mathbf{q}, t) \mathrm{d} q_{i}+A_{l}(\mathbf{q}, t) \mathrm{d} t=0 \quad l=1, \ldots, m
$$

Accordingly, the virtual displacement $\delta \mathbf{q}=\left[\delta q_{1}, \delta q_{2}, \ldots, \delta q_{n}\right]^{\mathrm{T}}$ is governed by

$$
\sum_{i=1}^{n} A_{l i}(\mathbf{q}, t) \delta q_{i}=0 \quad l=1, \ldots, m
$$

Suppose the system is under an impressed force $\mathbf{Q}=\left[Q_{1}, Q_{2}, \ldots, Q_{n}\right]^{\mathrm{T}}$. By letting $Q_{i}^{c}$, $i=1,2, \ldots, n$, denote the component of the constraint force in the direction $q_{i}$, the equation of motion of the constrained system, for each $i=1,2, \ldots, n$, is given by

$$
\frac{\mathrm{d}}{\mathrm{d} t} \frac{\partial T(\mathbf{q}, \dot{\mathbf{q}}, t)}{\partial \dot{q}_{i}}-\frac{\partial T(\mathbf{q}, \dot{\mathbf{q}}, t)}{\partial q_{i}}=Q_{i}(\mathbf{q}, \dot{\mathbf{q}}, t)+Q_{i}^{c}
$$

Since constraint equation (2.2) is ideal, by the principle of virtual work, the work done by the constraint force in every virtual displacement is zero, that is

$$
\mathbf{Q}^{c \mathrm{~T}} \delta \mathbf{q}=\sum_{i=1}^{n} Q_{i}^{c} \delta q_{i}=0
$$

The fundamental equation, from Lagrange's form of d'Alembert's principle (Yin et al., 2020), takes the form

$$
\sum_{i=1}^{n}\left(\frac{\mathrm{d}}{\mathrm{d} t} \frac{\partial T(\mathbf{q}, \dot{\mathbf{q}}, t)}{\partial \dot{q}_{i}}-\frac{\partial T(\mathbf{q}, \dot{\mathbf{q}}, t)}{\partial q_{i}}-Q_{i}(\mathbf{q}, \dot{\mathbf{q}}, t)\right) \delta q_{i}=0
$$

The Lagrange's equations of motion, after the use of the Principle of Relaxation of the Constraints (Hamel, 1949), for each $i=1,2, \ldots, n$, are given by

$$
\frac{\mathrm{d}}{\mathrm{d} t} \frac{\partial T(\mathbf{q}, \dot{\mathbf{q}}, t)}{\partial \dot{q}_{i}}-\frac{\partial T(\mathbf{q}, \dot{\mathbf{q}}, t)}{\partial q_{i}}-Q_{i}(\mathbf{q}, \dot{\mathbf{q}}, t)+\sum_{l=1}^{m} \lambda_{l} A_{l i}(\mathbf{q}, t)=0
$$

where $\lambda_{l}$ are the Lagrangian multipliers. The constraint force component $Q_{i}^{c}$ is related to the Lagrangian multiplier as

$$
Q_{i}^{c}=\sum_{l=1}^{m} \lambda_{l} A_{l i}(\mathbf{q}, t)=0 \quad i=1, \ldots, n
$$

The passive ideal constraint problem can be stated as follows: Given the initial condition of the state (i.e. $q\left(t_{0}\right)$ and $\dot{\mathbf{q}}\left(t_{0}\right)$, where $t_{0}$ is the initial time), determine $q_{i}(t), \dot{q}_{i}(t), i=1, \ldots, n$, and $\lambda_{i}(t), i=1, \ldots, m$, for all $t \geqslant t_{0}$. Note that this can be solved by uniting (2.3) and (2.8) which constitute a set of $n+m$ equations with $n+m$ variables $q_{1}, \ldots, q_{n}, \lambda_{1}, \ldots, \lambda_{m}$. It is worth realizing that the constraint force in (2.9) is to be decided after ideal constraint equation (2.2) is satisfied. That is, the surroundings of the system are able to implement this constraint force.

Remark. The nature of the passive ideal constraint problem is such that the designer is not responsible for actively generating the constraint force. It is generally assumed that the environment can automatically generate the required force so that the constraints are strictly obeyed. 


\section{Servo constraint problem}

Consider the mechanical system described above is following the constraints by a set of control forces rather than by the passive (i.e., material) environment. This kind of constraints are called servo constraints (also called prescribed constraints, program constraints, active constraints, or constraints of the second type in some other literature). Here, we call the control forces relative to the servo constraints the servo control. The equation of motion of original mechanical system (2.5) with the servo control, for each $i=1,2, \ldots, n$, can be rewritten as

$$
\frac{\mathrm{d}}{\mathrm{d} t} \frac{\partial T(\mathbf{q}, \dot{\mathbf{q}}, t)}{\partial \dot{q}_{i}}-\frac{\partial T(\mathbf{q}, \dot{\mathbf{q}}, t)}{\partial q_{i}}=Q_{i}(\mathbf{q}, \dot{\mathbf{q}}, t)+Q_{i, s}^{c}
$$

where $Q_{i}$ is the $i$-th impressed force (but not the servo controls), and $Q_{i, s}^{c}$ is the $i$-th constraint force provided by the active servo controls.

Based on the available controls, the structure of the constraint force is predetermined as

$$
Q_{i, s}^{c}=\sum_{l=1}^{p} u_{l} B_{i l}(\mathbf{q}, \dot{\mathbf{q}}, t)
$$

where $B_{i l}(\mathbf{q}, \dot{\mathbf{q}}, t) \in \mathbb{R}$ is determined by the structure of the servo controls that are available, and $u_{l} \in \mathbb{R}$ is the actual servo control.

Remark. The system is called redundant if the number of independent actuators is greater than the necessary to enforce servo constraints (i.e., $\operatorname{rank}\left[B_{i l}(\mathbf{q}, \dot{\mathbf{q}}, t)\right]>n$ ). On the other hand, called underactuated if the system has a lower number of actuators than its degrees of freedom, that is, $\operatorname{rank}\left[B_{i l}(\mathbf{q}, \dot{\mathbf{q}}, t)\right]<n$.

Suppose the system is to follow constraints (whether ideal or non-ideal) which are given by

$$
\sum_{i=1}^{n} A_{l i}(\mathbf{q}, t) \dot{q}_{i}+A_{l}(\mathbf{q}, t)=0 \quad l=1, \ldots, m
$$

where $m \geqslant 1, A_{l i}(\cdot)$ and $A_{l}(\cdot)$ are both $C^{1}$ in $q$ and $t$. These constraints are of the first order form as they imply restrictions on the velocities as well as the displacement.

We now convert the constraints into their second order form. Differentiating constraint equations (3.3) with respect to $t$ yields

$$
\sum_{i=1}^{n} \frac{\mathrm{d}}{\mathrm{d} t} A_{l i}(\mathbf{q}, t) \dot{q}_{i}+\sum_{i=1}^{n} A_{l i}(\mathbf{q}, t) \ddot{q}_{i}+\frac{\mathrm{d}}{\mathrm{d} t} A_{l}(\mathbf{q}, t)=0
$$

where

$$
\begin{aligned}
& \frac{\mathrm{d}}{\mathrm{d} t} A_{l i}(\mathbf{q}, t)=\sum_{k=1}^{n} \frac{\partial A_{l i}(\mathbf{q}, t)}{\partial q_{k}} \dot{q}_{k}+\frac{\partial A_{l i}(\mathbf{q}, t)}{\partial t} \\
& \frac{\mathrm{d}}{\mathrm{d} t} A_{l}(\mathbf{q}, t)=\sum_{k=1}^{n} \frac{\partial A_{l}(\mathbf{q}, t)}{\partial q_{k}} \dot{q}_{k}+\frac{\partial A_{l}(\mathbf{q}, t)}{\partial t}
\end{aligned}
$$

Equation (3.4), the second order form of the constraints, can be rewritten as

$$
\sum_{i=1}^{n} A_{l i}(\mathbf{q}, t) \ddot{q}_{i}=-\sum_{i=1}^{n} \frac{\mathrm{d}}{\mathrm{d} t} A_{l i}(\mathbf{q}, t) \dot{q}_{i}-\frac{\mathrm{d}}{\mathrm{d} t} A_{l}(\mathbf{q}, t)=b_{i}(\mathbf{q}, \dot{\mathbf{q}}, t)
$$

or, in a matrix form

$$
\mathbf{A}(\mathbf{q}, t) \ddot{\mathbf{q}}=\mathbf{b}(\mathbf{q}, \dot{\mathbf{q}}, t)
$$

where $\mathbf{A}=[A]_{m \times n}$ and $\mathbf{b}=\left[b_{1}, b_{2}, \ldots, b_{m}\right]^{\mathrm{T}}$. 
Remark. Chen (1999) stated that differentiation of the constraint to (3.7) will not result in the loss of information (such as an integration constant). The trajectory is determined by the equation of motion with the initial condition of $\mathbf{q}$ and $\dot{\mathbf{q}}$. This initial condition should satisfy the first order constraint (3.4). In other words, the missing information is in fact retained in the initial condition.

Remark. It has been demonstrated in (Xu et al., 2018) that various control problems, including stabilization, trajectory following and optimality, can be casted into the form of (3.7).

The servo constraint problem can now be stated as follows: Determine the servo control $u_{l}=u_{l}(\mathbf{q}, \dot{\mathbf{q}}, t), l=1, \ldots, p$, such that when $Q_{i, s}^{c}$ is applied to system equation (3.1), the resulting controlled system fulfills constraint (3.7).

\section{Moore-Penrose inverse}

We now supply the following mathematical preliminaries for later use (Noble and Daniel, 1977; Hui et al., 2018).

Definition. Consider a matrix $\Phi \in \mathbb{R}^{m \times n}$ with rank $r \leqslant 1$. Its singular values are given by $\sigma_{1} \geqslant \sigma_{2} \geqslant \cdots \geqslant \sigma_{r}>0$. Suppose that its singular-value decomposition is given by

$$
\mathbf{\Phi}=\mathbf{U} \boldsymbol{\Sigma} \mathbf{V}^{\mathrm{T}}=\sum_{i=1}^{r} \sigma_{i} u_{i} v_{i}^{\mathrm{T}}
$$

where $\boldsymbol{\Sigma}=\left[\operatorname{diag}\left(\sigma_{i}\right)\right]_{r \times r}$, both $\mathbf{U}=\left\{u_{1}, \ldots, u_{r}\right\} \in \mathbb{R}^{m \times r}$ and $\mathbf{V}=\left\{v_{1}, \ldots, v_{r}\right\} \in \mathbb{R}^{n \times r}$ are unitary matrices. Here $\left\{u_{1}, \ldots, u_{r}\right\}$ and $\left\{v_{1}, \ldots, v_{r}\right\}$ are orthonormal sets of vectors in $\mathbb{R}^{m}$ and $\mathbb{R}^{n}$, respectively. The Moore-Penrose (MP) inverse $\boldsymbol{\Phi}^{+} \in \mathbb{R}^{m \times n}$ of $\boldsymbol{\Phi}$ is given by

$$
\boldsymbol{\Phi}^{+}=\mathbf{V} \boldsymbol{\Sigma}^{-1} \mathbf{U}^{\mathrm{T}}
$$

Lemma. An $n \times m$ matrix $\mathbf{Y}$ is the MP inverse $\boldsymbol{\Phi}^{+}$of $\boldsymbol{\Phi}$ if and only if the following hold

$$
\begin{array}{lr}
\boldsymbol{\Phi} \mathbf{Y} \boldsymbol{\Phi}=\boldsymbol{\Phi} & \mathbf{Y} \boldsymbol{\Phi} \mathbf{Y}=\mathbf{Y} \\
(\boldsymbol{\Phi} \mathbf{Y})^{\mathrm{T}}=(\boldsymbol{\Phi} \mathbf{Y}) & (\mathbf{Y} \boldsymbol{\Phi})^{\mathrm{T}}=(\mathbf{Y} \boldsymbol{\Phi})
\end{array}
$$

Moreover, $\boldsymbol{\Phi}^{+}$is unique.

Lemma. Suppose $\boldsymbol{\Phi}$ can be decomposed as $\boldsymbol{\Phi}=\Psi_{1} \Psi_{2}$, where $\Psi_{1} \in \mathbb{R}^{m \times r}, \Psi_{2} \in \mathbb{R}^{r \times n}$. Suppose all three matrices have rank $r$. Then

$$
\boldsymbol{\Phi}^{+}=\Psi_{2}^{\mathrm{T}}\left(\Psi_{2} \Psi_{2}^{\mathrm{T}}\right)^{-1}\left(\Psi_{1}^{\mathrm{T}} \Psi_{1}\right)^{-1} \Psi_{1}^{\mathrm{T}}
$$

Theorem 1. Consider a matrix $\Phi \in \mathbb{R}^{m \times n}$ with rank $r \leqslant 1$. Then the following are true

(i) $\mathcal{R}\left(\boldsymbol{\Phi}^{\mathrm{T}}\right)=\mathcal{R}\left(\boldsymbol{\Phi}^{+}\right)=\mathcal{R}\left(\boldsymbol{\Phi}^{+} \boldsymbol{\Phi}\right)$

(ii) $\mathcal{N}(\boldsymbol{\Phi})=\mathcal{R}\left(\mathbf{I}-\boldsymbol{\Phi}^{+} \boldsymbol{\Phi}\right)$

where $\mathcal{R}(\cdot)$ (or $\mathcal{N}(\cdot)$ ) denotes the range (or null) space of the designated matrix. See the proof in (Chen, 2009).

Theorem 2. Consider a matrix $\boldsymbol{\Phi} \in \mathbb{R}^{m \times n}$. Any vector $\mathbf{y} \in \mathbb{R}^{n}$ can be decomposed into $\left(\mathbf{I}-\boldsymbol{\Phi}^{+} \boldsymbol{\Phi}\right) \mathbf{y}$, in $\mathcal{N}(\boldsymbol{\Phi})$, and $\boldsymbol{\Phi}^{+} \boldsymbol{\Phi} y$, in $\mathcal{R}(\boldsymbol{\Phi})$. The proof can be found in (Chen, 2009). 


\section{Mechanical system servo control design}

Substituting equation (2.1) into (3.1), the equation of motion of the mechanical system can be represented as

$$
\mathbf{M}(\mathbf{q}, t) \ddot{\mathbf{q}}+\left[\frac{\mathrm{d}}{\mathrm{d} t} \mathbf{M}(\mathbf{q}, t)\right] \dot{\mathbf{q}}+\frac{\mathrm{d} \mathbf{N}(\mathbf{q}, t)}{\mathrm{d} t}-\frac{\partial T(\mathbf{q}, \dot{\mathbf{q}}, t)}{\partial q}=\mathbf{Q}(\mathbf{q}, \dot{\mathbf{q}}, t)+\mathbf{Q}_{s}^{c}
$$

where $\mathbf{Q}_{s}^{c}=\left[Q_{1, s}^{c}, Q_{2, s}^{c}, \ldots, Q_{n, s}^{c}\right]^{\mathrm{T}}$. Denote $\boldsymbol{\tau}:=\mathbf{Q}_{s}^{c}$, mechanical system equation (5.1) can be organized in the commonly used form

$$
\mathbf{M}(\mathbf{q}, t) \ddot{\mathbf{q}}+\mathbf{C}(\mathbf{q}, \dot{\mathbf{q}}, t) \dot{\mathbf{q}}+\mathbf{g}(\mathbf{q}, t)+\mathbf{F}(\mathbf{q}, \dot{\mathbf{q}}, t)=\boldsymbol{\tau}
$$

Here $t \in \mathbb{R}$ is the independent variable, $q \in \mathbb{R}^{n}$ is the coordinate, $\dot{\mathbf{q}} \in \mathbb{R}^{n}$ is the velocity, $\ddot{\mathbf{q}} \in \mathbb{R}^{n}$ is the acceleration, and $\tau \in \mathbb{R}^{n}$ is the control input. Furthermore, $\mathbf{M}(\mathbf{q}, t) \in \mathbb{R}^{n \times n}$ is the inertial matrix, $C(\mathbf{q}, \dot{\mathbf{q}}, t) \dot{\mathbf{q}} \in \mathbb{R}^{n}$ is the Coriolis/centripetal force, $\mathbf{g}(\mathbf{q}, t) \in \mathbb{R}^{n}$ is the gravitational force, and $\mathbf{F}(\mathbf{q}, \dot{\mathbf{q}}, t) \in \mathbb{R}^{n}$ is the impressed force.

Assumption 1. For each $(\mathbf{q}, t) \in \mathbb{R}^{n} \times \mathbb{R}, \mathbf{M}(\mathbf{q}, t)>\mathbf{0}$.

In the past, it was often believed that the inertia matrix $\mathbf{M}(\mathbf{q}, t)$ was positive definite, and it was therefore considered a fact rather than an assumption. However, this belief has been shown to be untrue in (Chen et al., 1998).

Suppose the mechanical system is to follow $m$ holonomic or nonholonomic equality servo constraints of the form

$$
f_{i}(\mathbf{q}, \dot{\mathbf{q}}, t)=0 \quad i=1,2, \ldots, m
$$

Then by use of equation (3.4), we get a set of $m$ equations that are linear in $\ddot{\mathbf{q}}$, in the matrix form

$$
\mathbf{A}(\mathbf{q}, t) \ddot{\mathbf{q}}=\mathbf{b}(\mathbf{q}, \dot{\mathbf{q}}, t)
$$

Definition. For given $\mathbf{A}$ and $\mathbf{b}$, constraint equation (5.4) is said to be consistent if there exists at least one solution $\ddot{\mathbf{q}}$.

Assumption 2. Considering constraint equation (5.4), we assume that for each $(\mathbf{q}, t) \in \mathbb{R}^{n} \times \mathbb{R}$, $\operatorname{rank} \mathbf{A}(\mathbf{q}, t) \geqslant 1$. Furthermore, the constraint is consistent (this assumption ensures that the MP inverse of $\mathbf{A}$ exists).

We multiply both sides of (5.2) by $\mathbf{M}^{-\frac{1}{2}}$, then we have (from now on, arguments of functions are sometimes omitted for brevity, when no confusions are likely to arise)

$$
\mathbf{M}^{\frac{1}{2}} \ddot{\mathbf{q}}+\mathbf{M}^{-\frac{1}{2}}(\mathbf{C} \dot{\mathbf{q}}+\mathbf{g}+\mathbf{F})=\mathbf{M}^{-\frac{1}{2}} \boldsymbol{\tau}
$$

By letting $\ddot{\mathbf{x}}:=\mathbf{M}^{\frac{1}{2}} \ddot{\mathbf{q}}, \mathbf{a}:=-\mathbf{M}^{-\frac{1}{2}}(\mathbf{C} \dot{\mathbf{q}}+\mathbf{g}+\mathbf{F}), \boldsymbol{\tau}=\mathbf{B u}$, the equation of motion of the system can then be represented as

$$
\ddot{\mathbf{x}}=\mathbf{a}+\mathbf{M}^{-\frac{1}{2}} \mathbf{B u}=: \mathbf{a}+\overline{\mathbf{B}} u,
$$

where $\overline{\mathbf{B}}=\mathbf{M}^{-\frac{1}{2}} \mathbf{B}, \mathbf{B}=\left[B_{i l}\right]_{n \times p}, \mathbf{u}=\left[u_{1}, \ldots, u_{p}\right]^{\mathrm{T}}$. Also, we can rewrite equation (5.4) as

$$
\mathrm{AM}^{-\frac{1}{2}} \mathbf{M}^{\frac{1}{2}} \ddot{\mathbf{q}}=\mathbf{b}
$$


The servo constraint problem is equivalent to designing $\mathbf{u}$ so that system equation (5.6) obeys the constraint

$\mathbf{D} \ddot{\mathbf{x}}=\mathbf{b}$

where $\mathbf{D}:=\mathbf{A M}^{-\frac{1}{2}}$.

Substituting equation (5.6) into (5.8), we get

$\mathbf{D}(\mathbf{a}+\overline{\mathbf{B}} \mathbf{u})=\mathbf{b}$

Let $\overline{\mathbf{b}}:=\mathbf{b}-\mathbf{D a}$, we have

$(\mathbf{D} \overline{\mathbf{B}}) \mathbf{u}=\overline{\mathbf{b}}$

where $\mathbf{u} \in \mathbb{R}^{m}$ is unknown. This can also be viewed as a constraint on $\mathbf{u}$.

Definition. System equation (5.6) is said to be servo constraint controllable with respect to a set $\Omega_{q} \times \Omega_{\dot{q}} \times \Omega_{t}$ if there is a control $\mathbf{u}$ such that the system under this control meets constraint equation (5.8) for all $(\mathbf{q}, \dot{\mathbf{q}}, t) \in \Omega_{q} \times \Omega_{\dot{q}} \times \Omega_{t}$.

Let

$$
\Psi=\left\{(\mathbf{q}, \dot{\mathbf{q}}, t) \in \Omega_{q} \times \Omega_{\dot{q}} \times \Omega_{t} \mid \quad \mathbf{b}(\mathbf{q}, \dot{\mathbf{q}}, t) \neq \mathbf{D}(\mathbf{q}, t) \mathbf{a}(\mathbf{q}, \dot{\mathbf{q}}, t)\right\}
$$

and

$$
\Psi^{c}=\Omega_{q} \times \Omega_{\dot{q}} \times \Omega_{t} \backslash \Psi
$$

meaning that $\Psi \cup \Psi^{c}=\Omega_{q} \times \Omega_{\dot{q}} \times \Omega_{t}$.

Assumption 3. Considering $\mathbf{D}(\mathbf{q}, t), \overline{\mathbf{B}}(\mathbf{q}, \dot{\mathbf{q}}, t)$ and $\overline{\mathbf{b}}(\mathbf{q}, \dot{\mathbf{q}}, t)$, we assume that for each $(\mathbf{q}, \dot{\mathbf{q}}, t) \in \Psi$, equation (5.10) is consistent, and hence

$$
(\mathbf{D} \overline{\mathbf{B}})(\mathbf{D} \overline{\mathbf{B}})^{+} \overline{\mathbf{b}}=\overline{\mathbf{b}}
$$

which means that $\overline{\mathbf{b}} \in \mathcal{R}(\mathbf{D} \overline{\mathbf{B}})$.

Theorem 3 (Chen, 2009). Subject to Assumptions 1 to 3, the system equation is servo constraint controllable with respect to a set $\Omega_{q} \times \Omega_{\dot{q}} \times \Omega_{t}$ if and only if

$$
\operatorname{rank}[\mathbf{D}(\mathbf{q}, t) \overline{\mathbf{B}}(\mathbf{q}, \dot{\mathbf{q}}, t)] \geqslant 1
$$

for all $(\mathbf{q}, \dot{\mathbf{q}}, t) \in \Psi$. Furthermore, for all $(\mathbf{q}, \dot{\mathbf{q}}, t) \in \Psi$, the servo control $\mathbf{u}$ is given by

$$
\mathbf{u}=(\mathbf{D} \overline{\mathbf{B}})^{+} \overline{\mathbf{b}}+\left[\mathbf{I}-(\mathbf{D} \overline{\mathbf{B}})^{+}(\mathbf{D} \overline{\mathbf{B}})\right] \mathbf{S}
$$

where $\mathbf{S} \in \mathbb{R}^{m}$ is an arbitrary vector which may be dependent on $\dot{\mathbf{q}}$, q, and $t$. See in (Chen, 2009) for the detailed proof of sufficiency and necessity of the condition.

By Theorem 3, substituting $\mathbf{D}, \overline{\mathbf{B}}$, and $\overline{\mathbf{b}}$ into (5.15), the servo control we need to implement to the mechanical system is given by

$$
\mathbf{u}=\left(\mathbf{A M}^{-1} \mathbf{B}\right)^{+}\left[\mathbf{b}+\mathbf{A} \mathbf{M}^{-1}(\mathbf{C} \dot{\mathbf{q}}+\mathbf{g}+\mathbf{F})\right]+\left[\mathbf{I}-\left(\mathbf{A M}^{-1} \mathbf{B}\right)^{+}\left(\mathbf{A M}^{-1} \mathbf{B}\right)\right] \mathbf{S}
$$

where $\mathbf{S} \in \mathbb{R}^{m}$ is an arbitrary vector which makes the control more flexible. Suppose if the servo constraint is ideal and we choose $\mathbf{S}=\mathbf{0}$, then the second term on the right-hand side of the servo control $\mathbf{u}$ vanishes, only the first term leaves, which is the minimum effort for the servo control to fulfill the servo constraints. However, if the servo constraint is non-ideal, that is, some other forces such as the friction force or an electromagnetic force may come out and do work in the virtual displacement. Then we can design the vector $\mathbf{S}$ in the servo control to beat those forces. 
Remark. The servo control $\mathbf{u}$ consists of two general parts. The first term on the right-hand side of (5.16), which lies in the range space of the constraint surface, is the part of the control force to ensure that the constraint is exactly satisfied, while the second term on the right-hand side of (5.16) can be regarded as the part of the control force that will cause motion to the mechanical system. Hence, $\mathbf{S}$ is an optional vector which can be used to design motion of the system in the constraint surface. Also, the vector $\mathbf{S}$ can be designed to compensate the friction force, electromagnetic force or other force acting on the system.

Remark. The servo control $\mathbf{u}$ in equation (5.16) is a model based state feedback control, and is readily applicable. Note that the control is continuous in the state.

\section{Friction force depending on control design}

After servo control (5.16) is applied to the mechanical system, it is supposed to travel under the trajectory which can be described by servo constraint (5.3). In this Section, we will consider the non-ideal servo constraints case in which friction forces occur. It is very interesting to find out that when the constraints are non-ideal, the friction force and the control force are coupled.

To begin with, recalling that $\operatorname{rank}(\mathbf{A}) \geqslant 1$, by Theorems 2 and 3 , we decompose the control input $\boldsymbol{\tau}$ into two orthogonal vectors as (here we use $\boldsymbol{\tau}$ instead of $\mathbf{u}$ since it is the final servo control force acting on the system)

$$
\tau=\tau_{p}+\tau_{t}
$$

where

$$
\boldsymbol{\tau}_{p}=\mathbf{A}^{+} \mathbf{A} \boldsymbol{\tau} \quad \boldsymbol{\tau}_{t}=\left(\mathbf{I}-\mathbf{A}^{+} \mathbf{A}\right) \boldsymbol{\tau}
$$

Here $\mathbf{A}^{+} \mathbf{A} \boldsymbol{\tau} \in \mathcal{R}\left(\mathbf{A}^{\mathrm{T}}\right)$ is perpendicular to the constraint surface while $\left(\mathbf{I}-\mathbf{A}^{+} \mathbf{A}\right) \boldsymbol{\tau} \in \mathcal{N}(\mathbf{A})$ is tangent to the constraint surface. A geometric interpretation of equations (6.2) is shown in Fig. 1.

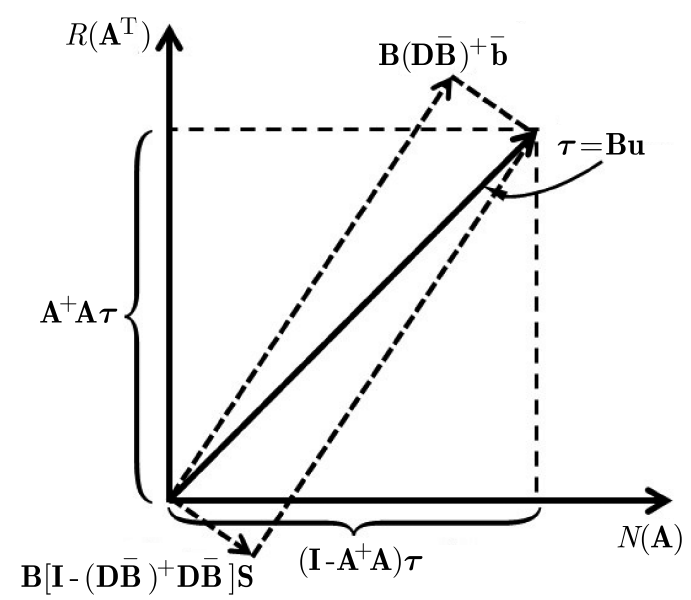

Fig. 1. Decomposition of the servo control force in the range space and null space of the constraint surface

Suppose the kinematic relations between the generalized coordinate and the Cartesian coordinate are as follows

$$
\begin{aligned}
& x=g_{x}\left(q_{1}, q_{2}, \ldots, q_{n}, t\right) \\
& y=g_{y}\left(q_{1}, q_{2}, \ldots, q_{n}, t\right) \\
& z=g_{z}\left(q_{1}, q_{2}, \ldots, q_{n}, t\right)
\end{aligned}
$$


Consider $\mathbf{J}$ is the matrix between the generalized coordinate and the Cartesian coordinate, which can be derived from equations (6.3) as

$$
\mathbf{J}=\left[\begin{array}{llll}
\frac{\partial g_{x}}{\partial q_{1}} & \frac{\partial g_{x}}{\partial q_{2}} & \cdots & \frac{\partial g_{x}}{\partial q_{n}} \\
\frac{\partial g_{y}}{\partial q_{1}} & \frac{\partial g_{y}}{\partial q_{2}} & \cdots & \frac{\partial g_{y}}{\partial q_{n}} \\
\frac{\partial g_{z}}{\partial q_{1}} & \frac{\partial g_{z}}{\partial q_{2}} & \cdots & \frac{\partial g_{z}}{\partial q_{n}}
\end{array}\right]
$$

Then we have

$$
\begin{aligned}
& \mathbf{F}_{n}=\left(\mathbf{J J}^{\mathrm{T}}\right)^{-1} \mathbf{J} \boldsymbol{\tau}_{p}=\left(\mathbf{J J J}^{\mathrm{T}}\right)^{-1} \mathbf{J} \mathbf{A}^{+} \mathbf{A} \boldsymbol{\tau} \\
& \mathbf{F}_{t}=\left(\mathbf{J J}^{\mathrm{T}}\right)^{-1} \mathbf{J} \boldsymbol{\tau}_{t}=\left(\mathbf{J J}^{\mathrm{T}}\right)^{-1} \mathbf{J}\left(\mathbf{I}-\mathbf{A}^{+} \mathbf{A}\right) \boldsymbol{\tau}
\end{aligned}
$$

Here $\mathbf{F}_{n}$ and $\mathbf{F}_{t}$ are the normal and tangent forces of the servo control $\boldsymbol{\tau}$ decomposed into the constraint surface in the Cartesian coordinate.

Suppose the system is under influence of the Coulomb friction force which is directly proportional to the normal force in magnitude and opposite to the direction of motion. Therefore, the expression of the Coulomb friction force $\mathbf{F}_{f}$ acting on the system may be represented as

$$
\mathbf{F}_{f}=-\mu\left\|\mathbf{F}_{n}\right\| \widehat{\mathbf{p}}(t) \quad \text { as } \quad \widehat{\mathbf{p}} \neq \mathbf{0}
$$

where $\mu$ is the friction coefficient in the corresponding directions, $\mathbf{F}_{n}$ is from $(6.5)_{1}$, and

$$
\widehat{\mathbf{p}}(t)=\frac{\mathbf{J}(\mathbf{q}(t), t) \dot{\mathbf{q}}(t)}{\|\mathbf{J}(\mathbf{q}(t), t) \dot{\mathbf{q}}(t)\|} \quad \text { as } \quad \dot{\mathbf{q}} \neq \mathbf{0}
$$

is the unit vector representing the motion direction in terms of the Jacobian.

Thus, the friction force that is coupled with the control force is given by

$$
\mathbf{F}_{f}=-\mu\left\|\left(\mathbf{J J}^{\mathrm{T}}\right)^{-1} \mathbf{J A}^{+} \mathbf{A} \boldsymbol{\tau}\right\| \frac{\mathbf{J} \dot{\mathbf{q}}}{\|\mathbf{J} \dot{\mathbf{q}}\|} \quad \text { as } \quad \mathbf{J} \dot{\mathbf{q}} \neq \mathbf{0}
$$

Remark. The magnitude of the friction force depends on $\mathbf{F}_{n}$, which depends on $\mathbf{q}, \dot{\mathbf{q}}$ and $t$. This dependence is based on the constraints (in terms of $\mathbf{A}$ and $\mathbf{b}$ ) as well as the control force. In other words, the expression of $\mathbf{F}_{n}$ is based on the constraints and control. For different controls, this expression needs to be rewritten. This is a surprising outcome, which defies some conventional wisdom in which it was (mistakenly) believed that the Coulomb friction force can be determined a priori of the control design. The current finding shows that the Coulomb friction force is based on the specific control applied. The (unknown) dependence of $\mathbf{F}_{n}$ on $\boldsymbol{\tau}$ is the major difficulty to achieve a closed-form expression of the Coulomb friction for a controlled mechanical system.

\section{Test case: A 3-DOF manipulator}

Consider a robot manipulator with three degrees of freedom (DOF) as shown in Fig. 2. The manipulator consists of three parts: the base, the first arm and the second arm, with mass $m_{1}$, $m_{2}, m_{3}$ and inertia moment $I_{1}, I_{2}, I_{3}$, respectively. Denote the directions of the space-fixed axes by $\widehat{\mathbf{I}}, \widehat{\mathbf{J}}, \widehat{\mathbf{K}}$ which are rigidly attached to an inertial reference frame (henceforth the inertial coordinate system). Denote $\left(\psi, \theta_{1}, \theta_{2}\right)$ as the joint coordinates of the base, the first arm and the second arm, respectively. Use $\left(\widehat{i}_{2}, \widehat{j}_{2}, \widehat{k}_{2}\right)$ and $\left(\hat{i}_{3}, \widehat{j}_{3}, \widehat{k}_{3}\right)$ as body-fixed coordinates to denote the position and orientation of the first and second arms. The base rolls are with angular velocity 
$\boldsymbol{\omega}_{1}=\dot{\psi} \widehat{\mathbf{K}}$ about the vertical axis $\widehat{\mathbf{K}}$. The first arm rotates about the base with angular velocity $\widehat{\boldsymbol{\omega}}_{2}=\dot{\theta}_{1} \widehat{\mathbf{i}}_{2}$ and the second arm rotates about the first arm with angular velocity $\widehat{\boldsymbol{\omega}}_{3}=\dot{\theta}_{2} \widehat{\mathbf{i}}_{3}$. Thus, the first arm and the second arm rotates with angular velocity $\boldsymbol{\omega}_{2}=\dot{\psi} \hat{\mathbf{K}}+\dot{\theta}_{1} \hat{\mathbf{i}}_{2}$ and $\boldsymbol{\omega}_{3}=\dot{\psi}_{i} \widehat{\mathbf{K}}+\dot{\theta}_{1} \widehat{\hat{\mathbf{i}}}_{2}+\dot{\theta}_{2} \widehat{\hat{i}}_{3}$ relative to the inertial frame, respectively. Assume the length of the first and the second arm are $L_{1}$ and $L_{2}$, respectively. Then the translational velocity of the second arm relative to the inertial frame is $\mathbf{v}_{3}=\boldsymbol{\omega}_{2} \times L_{2} \widehat{\mathbf{j}}_{2}+\boldsymbol{\omega}_{3} \times\left(L_{3} / 2\right) \widehat{\mathbf{j}}_{3}$.

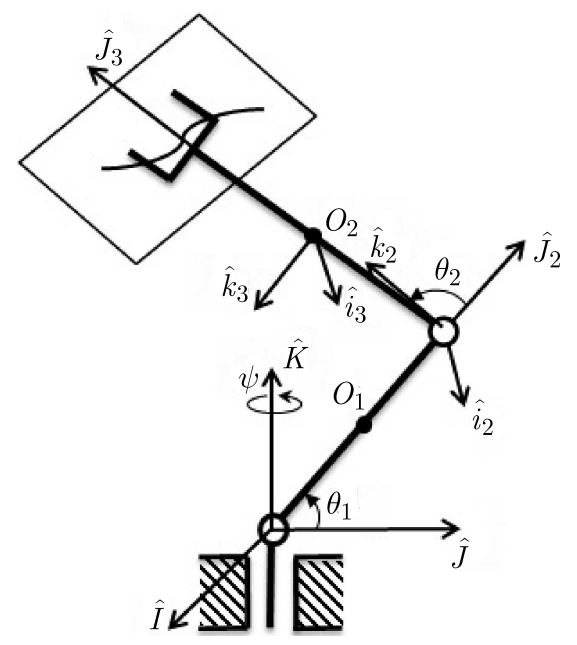

Fig. 2. A 3-DOF robot manipulator working under the servo constraint surface

By (2.8), we obtain the equation of motion of the manipulator and rewrite it in form of (5.2) as

$$
\underbrace{\left[\begin{array}{ccc}
m_{11} & 0 & 0 \\
0 & m_{22} & m_{23} \\
0 & m_{32} & m_{33}
\end{array}\right]}_{\mathbf{M}} \underbrace{\left[\begin{array}{c}
\ddot{\psi} \\
\ddot{\theta}_{1} \\
\ddot{\theta}_{2}
\end{array}\right]}_{\ddot{\mathbf{q}}}+\underbrace{\left[\begin{array}{lll}
c_{11} & c_{12} & c_{13} \\
c_{21} & c_{22} & c_{23} \\
c_{31} & c_{32} & c_{33}
\end{array}\right]}_{\mathbf{C}} \underbrace{\left[\begin{array}{c}
\dot{\psi} \\
\dot{\theta}_{1} \\
\dot{\theta}_{2}
\end{array}\right]}_{\dot{\mathbf{q}}}+\underbrace{\left[\begin{array}{c}
g_{1} \\
g_{2} \\
g_{3}
\end{array}\right]}_{\mathbf{g}}=\underbrace{\left[\begin{array}{c}
\tau_{1} \\
\tau_{2} \\
\tau_{3}
\end{array}\right]}_{\boldsymbol{\tau}}
$$

where

$$
\begin{aligned}
& m_{11}=I_{z 1}+I_{y 2} \sin \theta_{1}^{2}+I_{z 2} \cos \theta_{1}^{2}+m_{3}\left(L_{2} \cos \theta_{1}+\frac{L_{3}}{2} \cos \left(\theta_{1}+\theta_{2}\right)\right)^{2} \\
& \quad+I_{y 3} \sin \left(\theta_{1}+\theta_{2}\right)^{2}+I_{z 3} \cos \left(\theta_{1}+\theta_{2}\right)^{2} \\
& m_{22}=I_{x 2}+m_{3} L_{2}^{2} \sin \theta_{2}^{2}+m_{3}\left(L_{2} \cos \theta_{2} L_{2} \cos \theta_{2}+\frac{L_{3}}{2}\right) \\
& \quad+m_{3} \frac{L_{3}}{2}\left(L_{2} \cos \theta_{2}+\frac{L_{3}}{2}\right)+I_{x 3} \\
& m_{23}=m_{32}=m_{3} \frac{L_{3}}{2}\left(L_{2} \cos \theta_{2}+\frac{L_{3}}{2}\right)+I_{x 3} \\
& m_{33}=m_{3}\left(\frac{L_{3}}{2}\right)^{2}+I_{x 3}
\end{aligned}
$$

and $I_{z 1}$ represents the inertia moment of the base, $I_{x 2}, I_{y 2}$ and $I_{z 2}$ represent the inertia moment of the first arm, $I_{x 3}, I_{y 3}$ and $I_{z 3}$ represent the inertia moment of the second arm.

Here $\boldsymbol{\tau}$ is the servo control we need to design to make sure that the constraint is exactly followed. The detailed expressions of $\mathbf{C}$ and $\mathbf{g}$ are omitted for simplicity.

The kinetic energy of the manipulator is given by

$$
T=T_{1}+T_{2}+T_{3}=\frac{1}{2} I_{1} \boldsymbol{\omega}_{1}^{2}+\frac{1}{2} I_{2} \boldsymbol{\omega}_{2}^{2}+\frac{1}{2} I_{3} \boldsymbol{\omega}_{3}^{2}+\frac{1}{2} m_{3} v_{3}^{2}
$$


The potential energy of the manipulator is

$$
V=m_{2} g \frac{L_{2}}{2} \sin \theta_{1}+m_{3} g\left(L_{2} \sin \theta_{1}+\frac{L_{3}}{2} \sin \left(\theta_{1}+\theta_{2}\right)\right)
$$

Suppose the end-effector of the robot manipulator is required to work under the following constraint surface

$$
y+z=\text { const }
$$

We have the relations

$$
\begin{aligned}
& x=-\left[L_{2} \cos \theta_{1}+L_{3} \cos \left(\theta_{1}+\theta_{2}\right)\right] \sin \psi \\
& y=\left[L_{2} \cos \theta 1+L_{3} \cos \left(\theta_{1}+\theta_{2}\right)\right] \cos \psi \\
& z=L_{2} \sin \theta_{1}+L_{3} \sin \left(\theta_{1}+\theta_{2}\right)
\end{aligned}
$$

Thus, constraint equation (7.5) can be rewritten in the form of joint coordinates as

$$
\left[L_{2} \cos \theta_{1}+L_{3} \cos \left(\theta_{1}+\theta_{2}\right)\right] \cos \phi+L_{2} \sin \theta_{1}+L_{3} \sin \left(\theta_{1}+\theta_{2}\right)=\text { const }
$$

Taking derivative of (7.7) twice with respect to time $t$, we get the constraint equation in the form of (5.4) as

$$
\underbrace{\left[a_{1}, a_{2}, a_{3}\right]}_{\mathbf{A}} \underbrace{\left[\begin{array}{c}
\ddot{\psi}_{\psi} \\
\ddot{\theta}_{1} \\
\ddot{\theta}_{2}
\end{array}\right]}_{\ddot{\mathbf{q}}}=\underbrace{b_{1}}_{\mathbf{b}}
$$

where

$$
\begin{aligned}
& a_{1}=-L_{2} \cos \theta_{1} \sin \psi-L_{3} \cos \left(\theta_{1}+\theta_{2}\right) \sin \psi \\
& a_{2}=-L_{2} \sin \theta_{1} \cos \psi-L_{3} \sin \left(\theta_{1}+\theta_{2}\right) \cos \psi+L_{2} \cos \theta_{1}+L_{3} \cos \left(\theta_{1}+\theta_{2}\right) \\
& a_{3}=-L_{3} \sin \left(\theta_{1}+\theta_{2}\right) \cos \psi+L_{3} \cos \left(\theta_{1}+\theta_{2}\right)
\end{aligned}
$$

and

$$
\begin{aligned}
b_{1}= & L_{2} \cos \theta_{1} \cos \psi \dot{\theta}_{1}^{2}-L_{2} \sin \theta_{1} \sin \psi \dot{\psi} \dot{\theta}_{1}-L_{2} \sin \theta_{1} \sin \psi \dot{\psi} \dot{\theta}_{1}+L_{2} \cos \theta_{1} \sin \psi \dot{\psi}^{2} \\
& +L_{3} \cos \left(\theta_{1}+\theta_{2}\right) \cos \psi\left(\dot{\theta}_{1}+\dot{\theta}_{2}\right)^{2}-L_{3} \sin \left(\theta_{1}+\theta_{2}\right) \sin \psi \dot{\psi}\left(\dot{\theta}_{1}+\dot{\theta}_{2}\right) \\
& -L_{3} \sin \left(\theta_{1}+\theta_{2}\right) \sin \psi \dot{\psi}\left(\dot{\theta}_{1}+\dot{\theta}_{2}\right)+L_{3} \cos \left(\theta_{1}+\theta_{2}\right) \cos \psi \dot{\psi}^{2}+L_{2} \sin \theta_{1} \dot{\theta}_{1}^{2} \\
& +L_{3} \sin \left(\theta_{1}+\theta_{2}\right)\left(\dot{\theta}_{1}+\dot{\theta}_{2}\right)^{2}
\end{aligned}
$$

Here, for simplicity, suppose we can control this 3-DOF robot manipulator by input torque on joint coordinates $\psi, \theta_{1}$, and $\theta_{2}$ independently. Then we have

$$
\mathbf{B}=\left[\begin{array}{lll}
1 & 0 & 0 \\
0 & 1 & 0 \\
0 & 0 & 1
\end{array}\right]
$$

By equation (5.16), as $\mathbf{B}$ is a unit matrix, the servo control force is thus

$$
\boldsymbol{\tau}=\left(\mathbf{A M}^{-1}\right)^{+}\left[\mathbf{b}+\mathbf{A} \mathbf{M}^{-1}(\mathbf{C} \dot{\mathbf{q}}+\mathbf{g})\right]+\left[\mathbf{I}-\left(\mathbf{A M}^{-1}\right)^{+}\left(\mathbf{A M}^{-1}\right)\right] \mathbf{S}
$$

where the first term on the right-hand side of (7.13) is a part of the control force to ensure that the constraint is satisfied, the second term on the right-hand side of (7.13) can be considered as 
a part of the control force that will cause motion to the robot manipulator. Hence, $\mathbf{S}$ is a $3 \times 1$ optional vector which can be used to design motion of the manipulator in the constraint surface.

From equations (7.6) to (7.13), we get the Jacobian matrix

$$
\mathbf{J}=\left[\begin{array}{ccc}
-L_{2} \overline{\mathrm{c}} \theta_{1} \overline{\mathrm{c}} \psi-L_{3} \overline{\mathrm{c}}\left(\theta_{1}+\theta_{2}\right) \overline{\mathrm{c}} \psi & L_{2} \overline{\mathrm{s}} \theta_{1} \overline{\mathrm{s}} \psi+L_{3} \overline{\mathrm{s}}\left(\theta_{1}+\theta_{2}\right) \overline{\mathrm{s}} \psi & L_{3} \overline{\mathrm{s}}\left(\theta_{1}+\theta_{2}\right) \overline{\mathrm{s}} \psi \\
-L_{2} \overline{\mathrm{c}} \theta_{1} \overline{\mathrm{s}} \psi-L_{3} \overline{\mathrm{c}}\left(\theta_{1}+\theta_{2}\right) \overline{\mathrm{s}} \psi & -L_{2} \overline{\mathrm{s}} \theta_{1} \overline{\mathrm{c}} \psi-L_{3} \overline{\mathrm{s}}\left(\theta_{1}+\theta_{2}\right) \overline{\mathrm{c}} \psi & -L_{3} \overline{\mathrm{s}}\left(\theta_{1}+\theta_{2}\right) \overline{\mathrm{c}} \psi \\
0 & L_{2} \overline{\mathrm{c}} \theta_{1}+L_{3} \overline{\mathrm{c}}\left(\theta_{1}+\theta_{2}\right) & L_{3} \overline{\mathrm{c}}\left(\theta_{1}+\theta_{2}\right)
\end{array}\right]
$$

here $\overline{\mathrm{s}}$ denotes sin, $\overline{\mathrm{c}}-\cos$.

By equation (6.8), the friction force coupled with the control force is given by

$$
\mathbf{F}_{f}=-\mu\left\|\left(\mathbf{J J}^{\mathrm{T}}\right)^{-1} \mathbf{J A}^{+} \mathbf{A} \boldsymbol{\tau}\right\| \frac{\mathbf{J} \dot{\mathbf{q}}}{\|\mathbf{J} \dot{\mathbf{q}}\|} \quad \text { as } \quad \mathbf{J} \dot{\mathbf{q}} \neq \mathbf{0}
$$

\section{Numerical simulation results}

Assume the robot manipulator with the unit mass $m_{1}=m_{2}=m_{3}=1$ and unit arm length $l_{1}=l_{2}=l_{3}=1$, and for simplicity, choose moments of inertia of the base, the first and the second arm are all unit $I_{z 1}=I_{x 2}=I_{y 2}=I_{z 2}=I_{x 3}=I_{y 3}=I_{z 3}=1$. Suppose the end-effector of the robot manipulator is going to move under a constraint surface which can be described by $y+z=2.59$. Choose the gravitational acceleration $g=9.8$ and the friction coefficient $\mu=0.1$. Consider the initial position of the joint coordinates are $\psi=0, \theta_{1}=\theta_{2}=\pi / 12$ while the initial velocities are all zero. We select three different types of the control force by changing the vector $\mathbf{S}$ in equation (7.13) as follows

$$
\mathbf{S}_{1}=[0,0,0]^{\mathrm{T}} \quad \mathbf{S}_{2}=[1,2,3]^{\mathrm{T}} \quad \mathbf{S}_{3}=[\sin 2 t, \cos 2 t, \sin 2 t]^{\mathrm{T}}
$$

where $\mathbf{S}_{1}, \mathbf{S}_{2}$, and $\mathbf{S}_{3}$ are zero, constant and time-varying vectors.

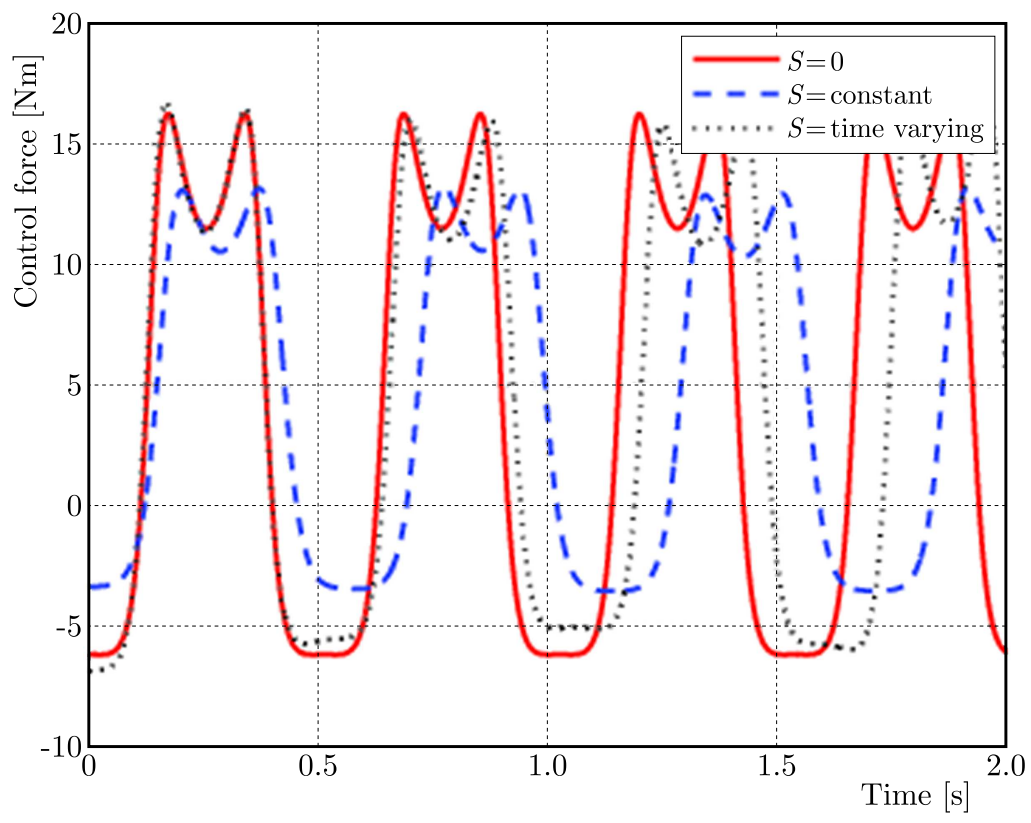

Fig. 3. The control force exerting from the joint coordinates of the base under three different control forces where $\mathbf{S}=[0,0,0 t]^{\mathrm{T}}, \mathbf{S}=[1,2,3]^{\mathrm{T}}$ and $\mathbf{S}=[\sin 2 t, \cos 2 t, \sin 2 t]^{\mathrm{T}}$, respectively

By following the modeling procedures of the robot manipulator illustrated in the previous Section and coding the equations in Matlab, we get a corresponding simulation result. Figures 


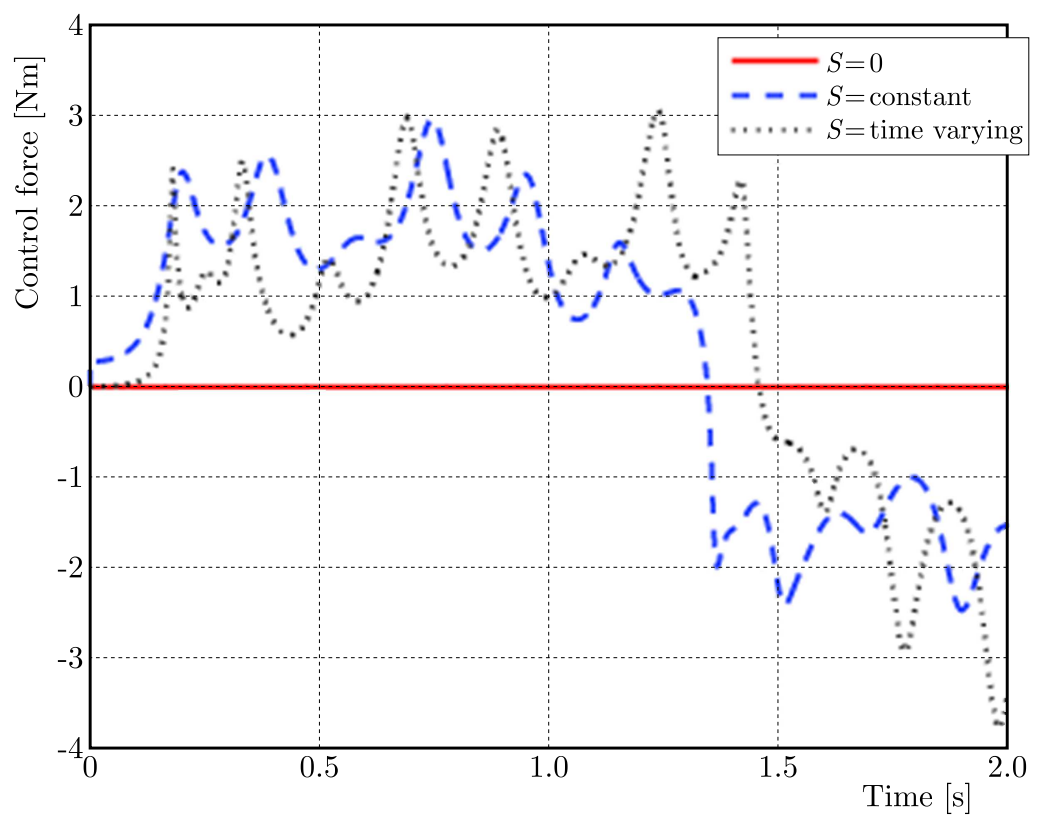

Fig. 4. The control force exerting from the joint coordinates of the first arm under three different control forces where $\mathbf{S}=[0,0,0]^{\mathrm{T}}, \mathbf{S}=[1,2,3]^{\mathrm{T}}$ and $\mathbf{S}=[\sin 2 t, \cos 2 t, \sin 2 t]^{\mathrm{T}}$, respectively

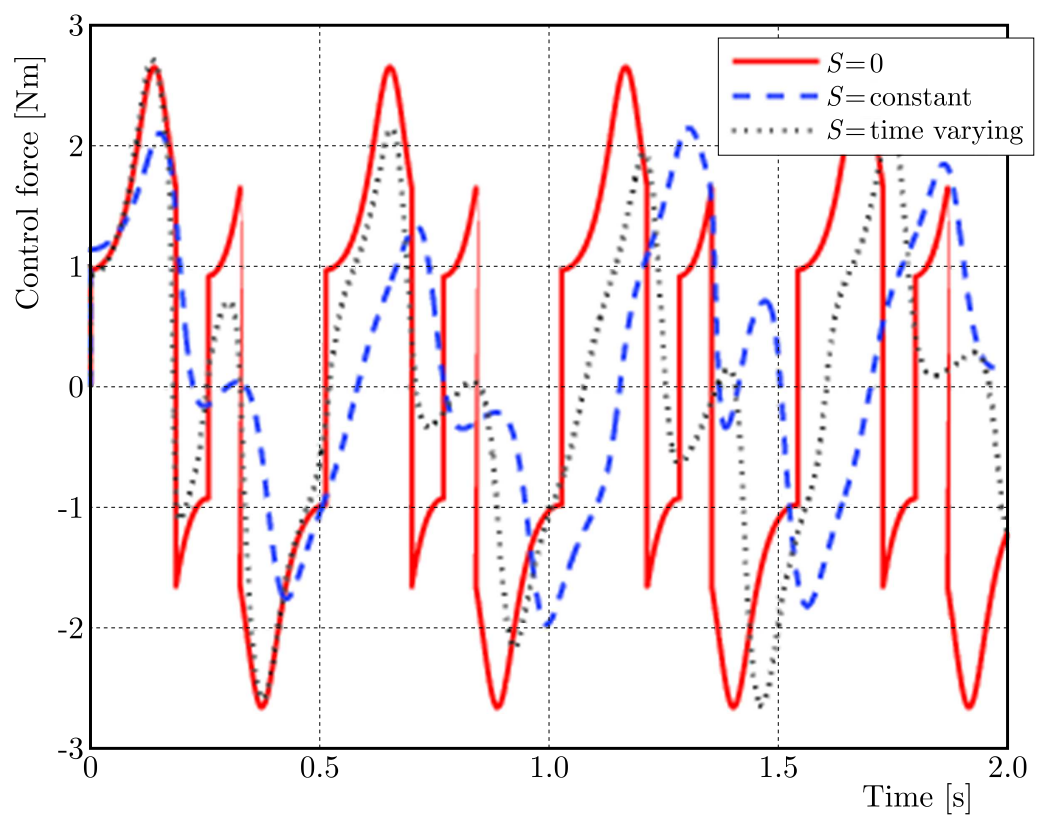

Fig. 5. The control force exerting from the joint coordinates of the second arm under three different control forces where $\mathbf{S}=[0,0,0]^{\mathrm{T}}, \mathbf{S}=[1,2,3]^{\mathrm{T}}$ and $\mathbf{S}=[\sin 2 t, \cos 2 t, \sin 2 t]^{\mathrm{T}}$, respectively

3-5 show the control force needed to apply at the joint coordinates of the base, the first arm, and the second arm under three different control forces, respectively.

These control forces are $\boldsymbol{\tau}$ which will cause the end-effector to move on the constraint surface. Figures 6-8 show the related friction forces exerting at the end-effector along with the $x, y, z$ directions under the three types of control forces. We can see from these figures that the contrast of the friction forces is remarkable under different controls. Thus the friction forces and the control forces are coupled. 


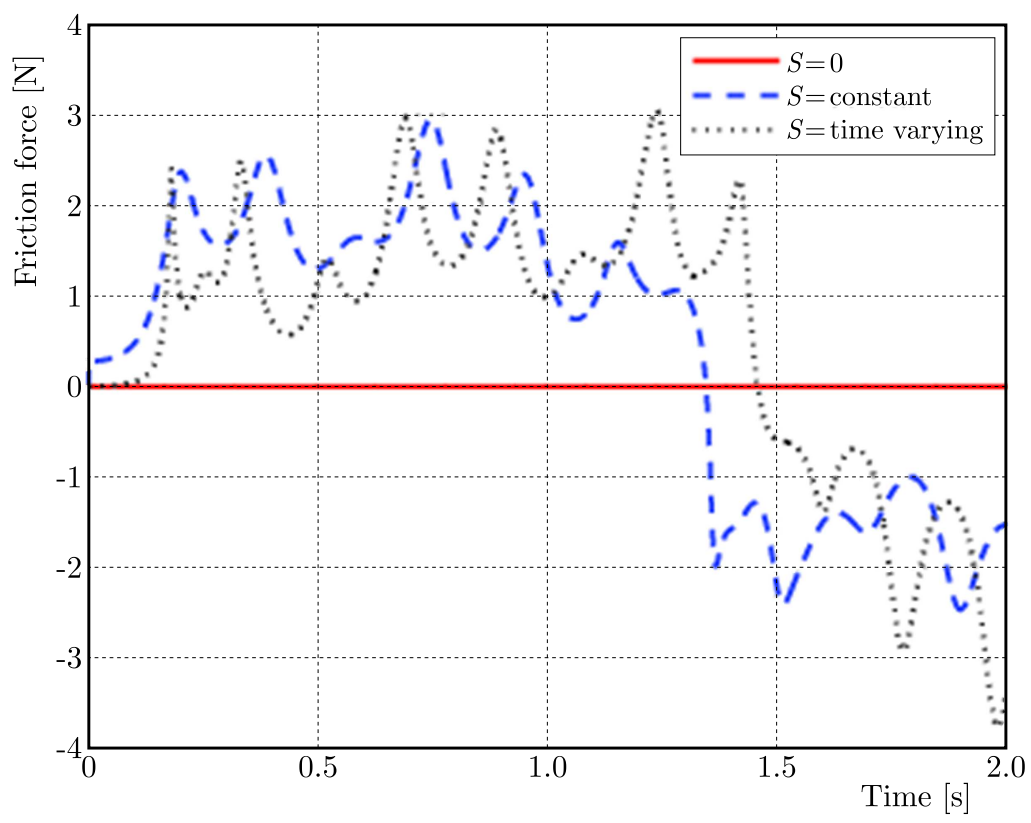

Fig. 6. The friction forces generated along the $x$ direction under three different control forces where $\mathbf{S}=[0,0,0]^{\mathrm{T}}, \mathbf{S}=[1,2,3]^{\mathrm{T}}$ and $\mathbf{S}=[\sin 2 t, \cos 2 t, \sin 2 t]^{\mathrm{T}}$, respectively

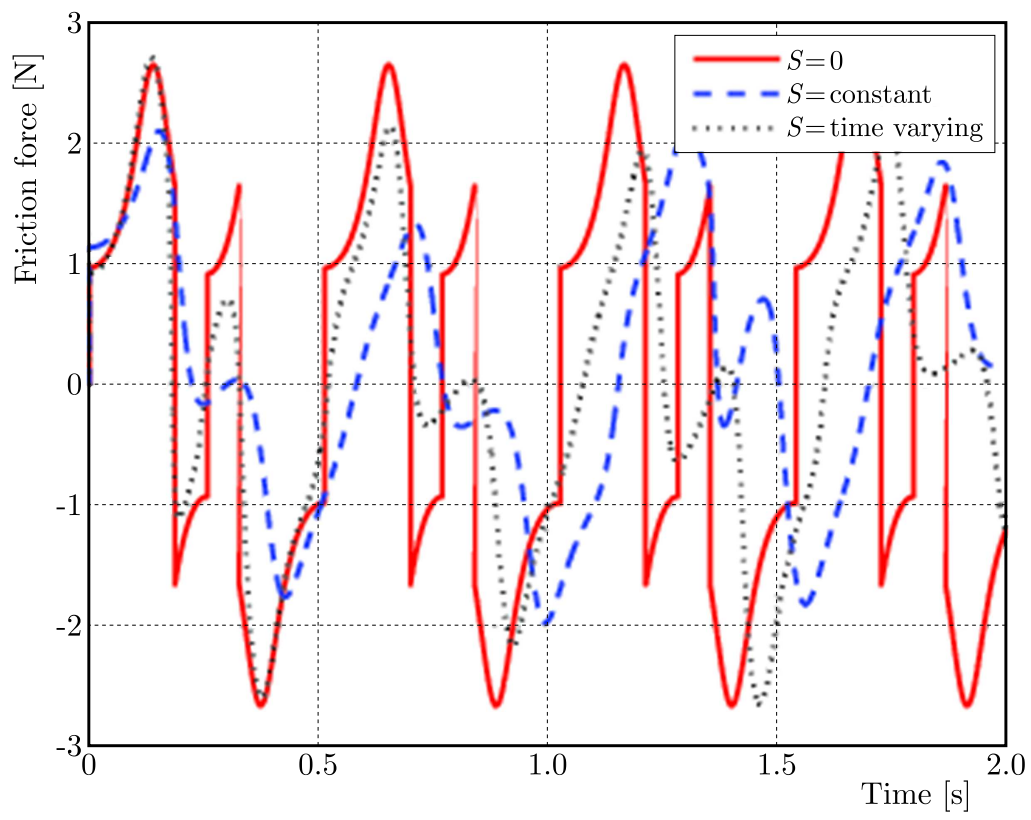

Fig. 7. The friction forces generated along the $y$ direction under three different control forces where $\mathbf{S}=[0,0,0]^{\mathrm{T}}, \mathbf{S}=[1,2,3]^{\mathrm{T}}$ and $\mathbf{S}=[\sin 2 t, \cos 2 t, \sin 2 t]^{\mathrm{T}}$, respectively

Figures 9-11 show the angular displacement of the base, the first and second arm of the robot manipulator under three different types of the control force, respectively. And Fig. 12 shows the constraint following error that the end-effector deviates from the constraint surface. We can see that the error is really small and the constraint is almost exactly followed under this control input. 


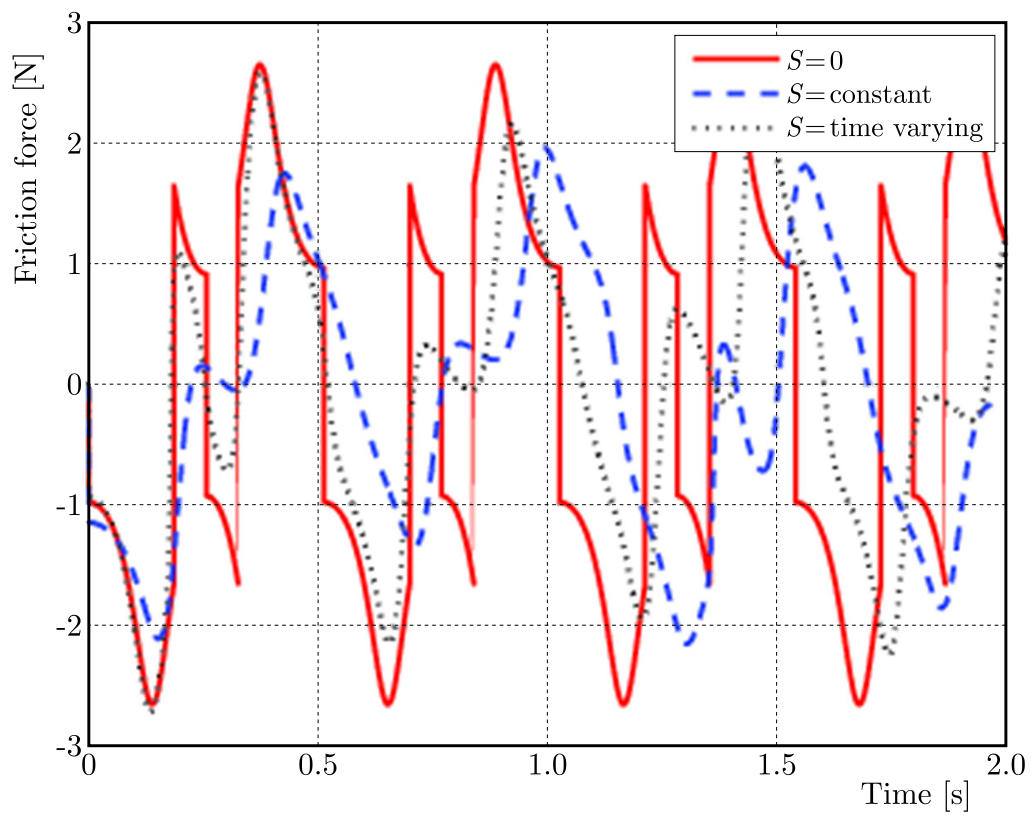

Fig. 8. The friction forces generated along the $z$ direction under three different control forces where $\mathbf{S}=[0,0,0]^{\mathrm{T}}, \mathbf{S}=[1,2,3]^{\mathrm{T}}$ and $\mathbf{S}=[\sin 2 t, \cos 2 t, \sin 2 t]^{\mathrm{T}}$, respectively

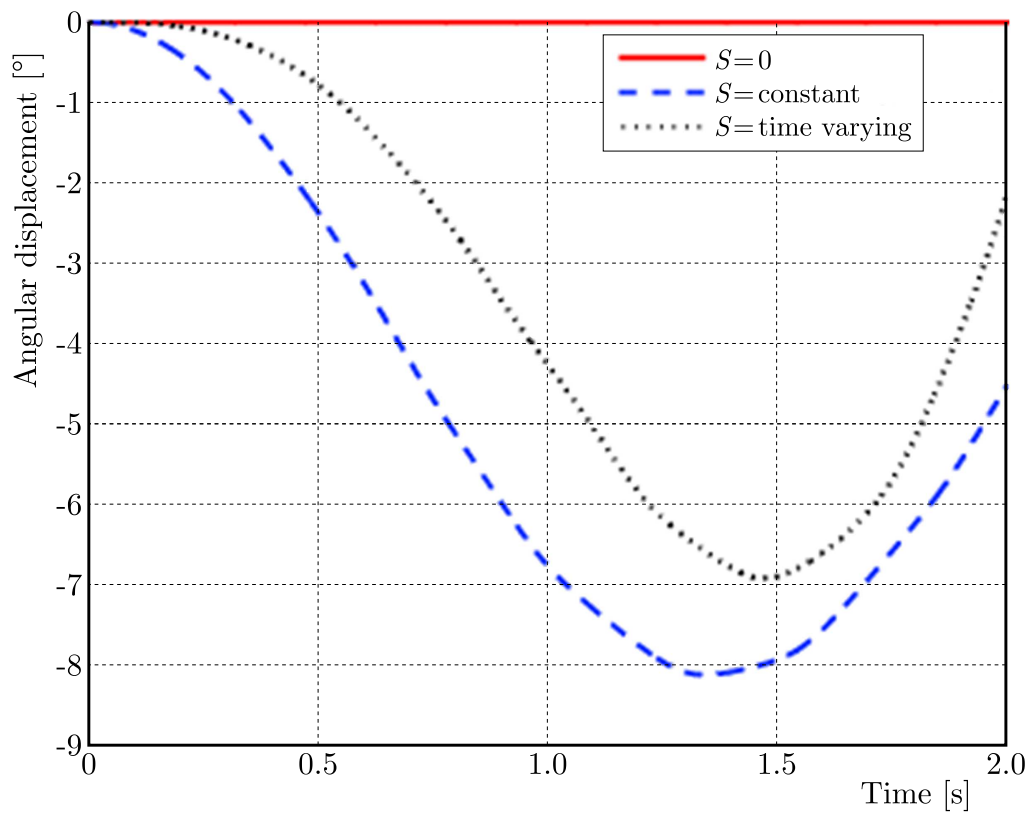

Fig. 9. The angular displacement of the base under three different control forces where $\mathbf{S}=[0,0,0]^{\mathrm{T}}$, $\mathbf{S}=[1,2,3]^{\mathrm{T}}$ and $\mathbf{S}=[\sin 2 t, \cos 2 t, \sin 2 t]^{\mathrm{T}}$, respectively

\section{Conclusions}

A robot manipulator is often taken to perform tasks repeatedly with utmost accuracy as a substitute to a human arm. The analytical friction force is crucial for the robot manipulator control design if one wants to get an accurate constraint following control. In the past, the friction force is supposed to be compensated by a certain control strategy. In this paper, however, we have shown that the friction force may vary with the control input, i.e., they are coupled. Then method of servo control has been introduced and applied to the constraint following control of robot manipulators. The second order constraint is developed so that the constraint equation 


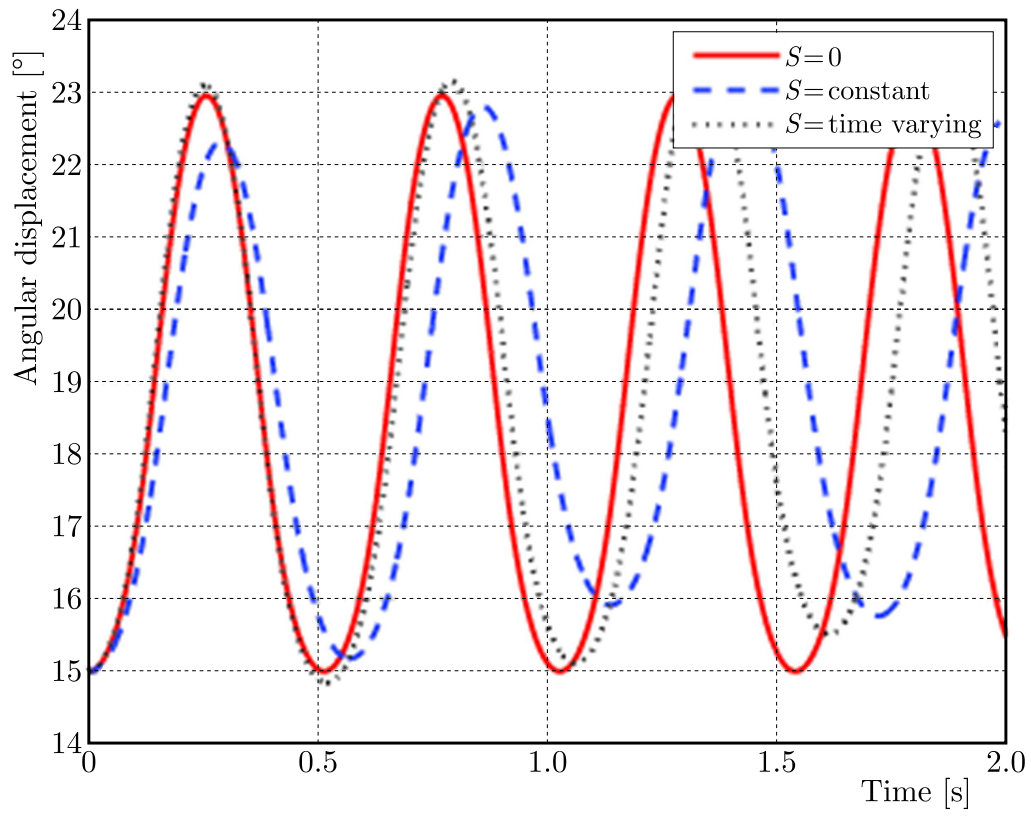

Fig. 10. The angular displacement of the first arm under three different control forces where $\mathbf{S}=[0,0,0]^{\mathrm{T}}, \mathbf{S}=[1,2,3]^{\mathrm{T}}$ and $\mathbf{S}=[\sin 2 t, \cos 2 t, \sin 2 t]^{\mathrm{T}}$, respectively

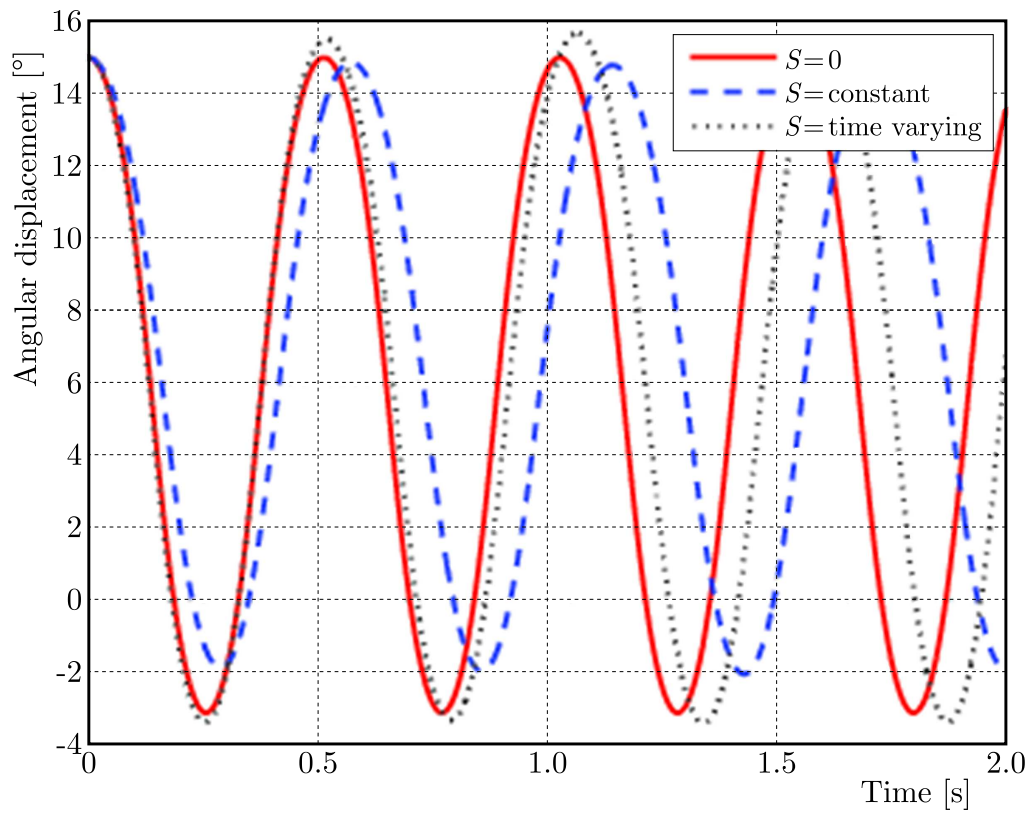

Fig. 11. The angular displacement of the second arm under three different control forces where $\mathbf{S}=[0,0,0]^{\mathrm{T}}, \mathbf{S}=[1,2,3]^{\mathrm{T}}$ and $\mathbf{S}=[\sin 2 t, \cos 2 t, \sin 2 t]^{\mathrm{T}}$, respectively

can be written in a united form for both holonomoic and nonholonomic constraints. By virtue of this constraint, the control input is derived in a closed-form, and so does the friction force related to the control forces. The control input consists of two terms, the first term is the forces that make the manipulators satisfy the constraints, while the second term can be designed to compensate gravitational forces or friction forces, etc. This will be really helpful in the trajectory following control of robot manipulators. 


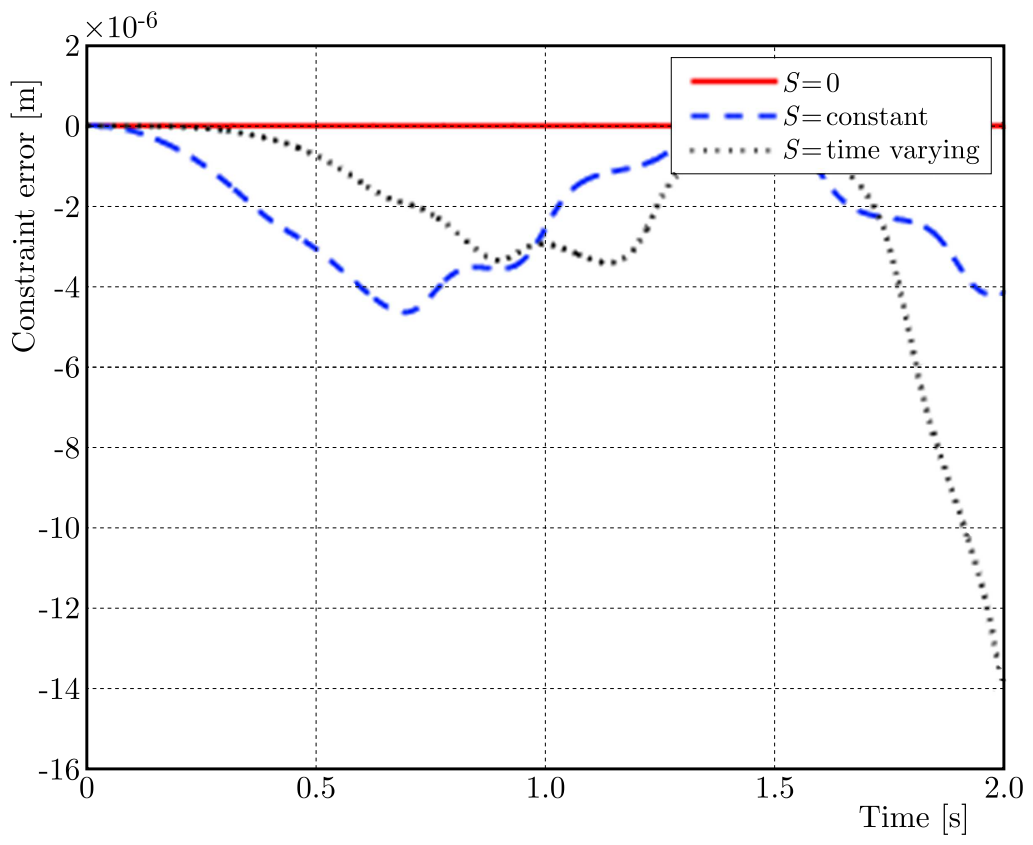

Fig. 12. The numerical constraint following error under three different control forces where $\mathbf{S}=[0,0,0]^{\mathrm{T}}, \mathbf{S}=[1,2,3]^{\mathrm{T}}$ and $\mathbf{S}=[\sin 2 t, \cos 2 t, \sin 2 t]^{\mathrm{T}}$, respectively

\section{References}

1. Abraham R., Marsden J.E., Ratiu T., 1988, Tensor Analysis, and Applications, 2nd ed., Springer Verlag, New York

2. Anderson B.D.O., Moore J.B., 1969, Linear system optimization with prescribed degree of stability, Proceedings of IEEE, 116, 2083-2087

3. Cabannes H., 1968, General Mechanics, 2nd ed. (translated, original in French), Blaisdell/Ginn, Waltham, MA

4. Chen Y.H., 1999, Equations of motion of constrained mechanical systems: given force depends on constraint force, Mechatronics, 9, 4, 411-428

5. Chen Y.H., 2009, Constraint-following servo control design for mechanical system, Journal of Vibration and Control, 15, 3, 369-389

6. Chen Y.H., Leitmann G.H., Chen J.S., 1998, Robust control for rigid serial manipulators: A general setting, Proceedings of the 1998 American Control Conference, Philadelphia, PA, 2, 912-916

7. Hamel G., 1949, Theoretische Mechanik (in German), Springer Verlag, Berlin

8. Huang J., Chen Y.H., Zhong Z., 2013, Udwadia-Kalaba approach for parallel manipulator dynamics, Journal of Dynamic Systems Measurement and Control, 135, 6, 061003

9. Hui Y., Chen Y., Yu D., 2018, Vehicle motion control under equality and inequality constraints: a diffeomorphism approach, Nonlinear Dynamics, 95, 175-194

10. Kirgetov V.I., 1967, The motion of controlled mechanical systems with prescribed constraints (servoconstraints) (in English, translated from the original Russian), Journal of Applied Mathematics and Mechanics 31, 3, 433-446

11. Noble B., Daniel J.W., 1977, Applied Linear Algebra, 2nd ed., Prentice Hall, Englewood Cliffs, N.J.

12. Papastavridis J.G., 2002, Analytical Mechanics, Oxford University Press, New York, NY

13. Rosenberg R.M., 1977, Analytical Dynamics of Discrete Systems, Plenum, New York 
14. Sciavicco L., Siciliano B., 2000, Modeling and Control of Robot Manipulators, 2nd ed., Springer Verlag, UK

15. Sun H., Zhao H., Zhen S., Huang K., Zhao F., Chen X., Chen Y.-H., , 2015, Application of the Udwadia-Kalaba approach to tracking control of mobile robots, Nonlinear Dynamics, 83, 389-400

16. Udwadia F.E., Kalaba R.E., 1996, Analytical Dynamics: A New Approach, Cambridge University Press, Cambridge, UK

17. Udwadia F.E., Kalaba R.E., 2000, Nonideal constraints and Lagrangian dynamics, Journal of Aerospace Engineering, 13, 17-22

18. Xu J., Du Y., Chen Y.-H., Guo H., 2018, Adaptive robust constrained state control for nonlinear maglev vehicle with guaranteed bounded airgap, IET Control Theory and Applications, 12, 11, $1573-1583$

19. Yin H., Chen Y.-H., Huang J., Lü H., 2020, Tackling mismatched uncertainty in robust constraint-following control of under actuated systems, Information Sciences, 520, 337-352

20. Zhao R., Chen Y.H., Wu L., PAn M., 2020, Robust trajectory tracking control for uncertain mechanical systems: servo constraint-following and adaptation mechanism, International Journal of Control, 93, 7, 1696-1709

Manuscript received December 18, 2020; accepted for print April 15, 2021 